\section{Shape memory textiles for functional compression management}

\section{Bipin Kumar}

Institute of Textiles and Clothing, The Hong Kong Polytechnic University, Kowloon, Hung Hom, Hong Kong

Compression stockings or bandages are preferred choice for the management of venous ulcers and also prevention of the recurrence ulcers. ${ }^{1,2}$ Nevertheless, current textile based compression products have the following shortcomings: i) selection of stockings with proper sizing and fitting has always been a challenge for both health practitioners and manufacturers seeking better patient compliance and effective treatment; ii) using textiles, it is impossible to achieve a dynamic (massaging) compression benefit which is otherwise provided by intermittent pneumatic compression which is costly, noisy, bulky, and once attached, severely constraining the patients; iii) To apply a targeted compression or pressure level and its sustenance is a huge challenge to provide effective treatment. Moreover, pressure drop is inevitable for almost all available stockings, and the replacement of stocking is needed once the pressure fall below a target level.

This work aims to design and investigate smart textiles using memory polymer to overcome the existing challenges of the compression products. Smart fabric was developed by incorporating memory filaments in the fabric structure (Figure 1). ${ }^{3,4}$ The memory filament was used as inlay yarn in the structure and it was made from segmented polyurethane. Nylon was used as ground yarn in the structure.

The developed smart fabric allows to program customized shape development (Figure 2). This shape control ability of the memory textile will promote the development of one size stocking that could be programmed to fit different leg sizes. This will be a huge relief to doctor or manufactures in stocking selection as different size requirements could be met by just one memory stocking.

Apart from shape control, the memory
Correspondence: Bipin Kumar, Institute of Textiles and Clothing, The Hong Kong Polytechnic University, Kowloon, Hung Hom, Hong Kong.

E-mail: bipiniitd18@gmail.com

Acknowledgments: the author acknowledges the full guidance and support from his previous postdoc supervisors, i.e. Prof. Jinlian $\mathrm{Hu}$ and Prof. Ning Pan. Most of this research has been carried out at the Institute of Textiles and Clothing, The Hong Kong Polytechnic University, HK. The author also acknowledges the support of team members, Harishkumar Narayana, H. Jianping and Wu You for all discussion and experimentation.

This work is licensed under a Creative Commons Attribution 4.0 License (by-nc 4.0).

(C) Copyright B. Kumar, 2017

Licensee PAGEPress, Italy

Veins and Lymphatics 2017; 6:6633

doi:10.4081/vl.2017.6633

polymer also allows to control stress in the structure. ${ }^{5}$ As the interface pressure depends on the stress (tension) in the fabric material,

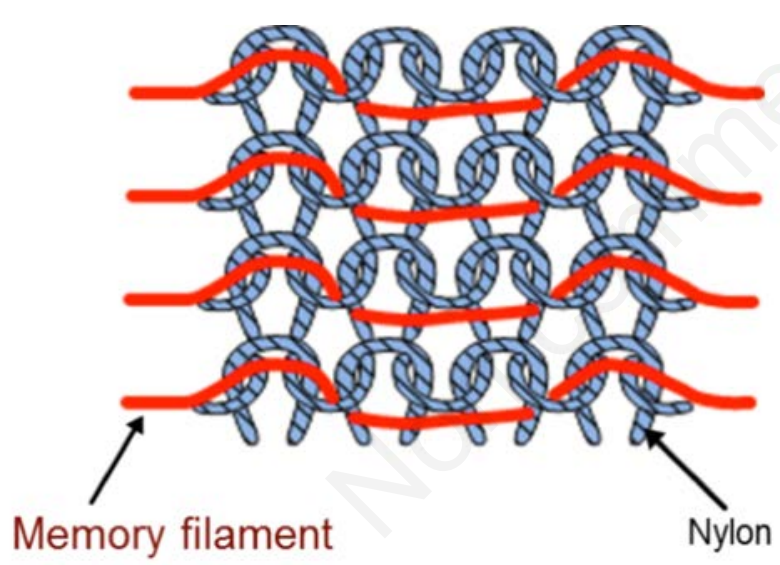

Figure 1. Smart fabric.

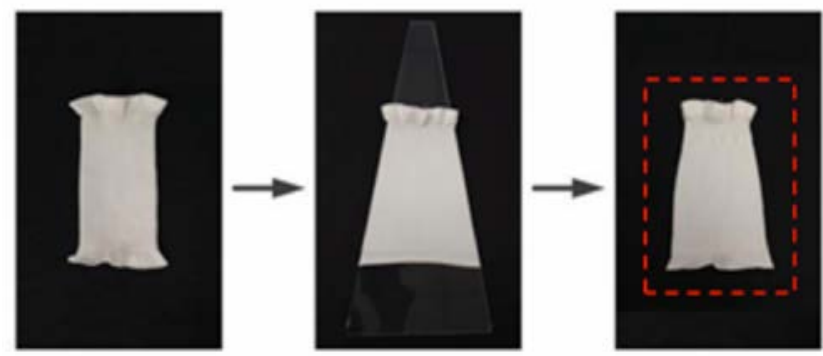

Figure 2. Customized shape development using the memory fabric.

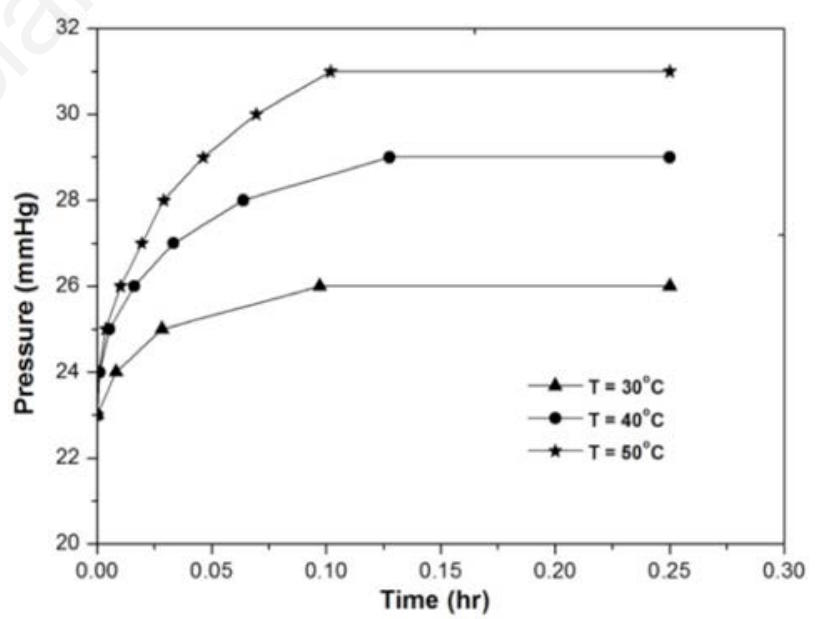

Figure 3. Customized shape development using the memory fabric.
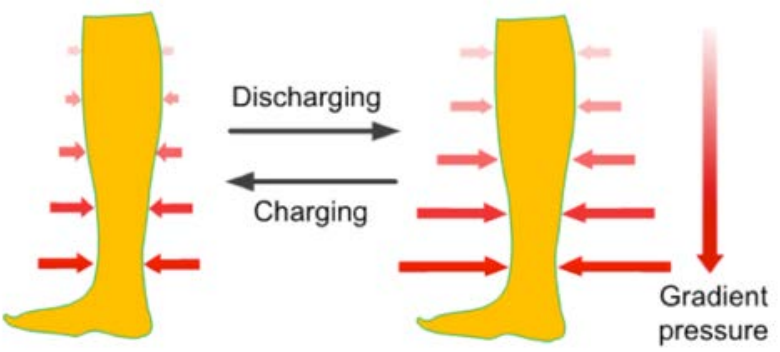

Figure 4. Schematic of memory fabric performance showing dynamic compression. 
so it is possible to control pressure by modulating internal stress in its structure. To confirm this, we tested the memory fabric performance in changing pressure. The pressure level can be changed by changing temperature (Figure 3).

Because of the internal stress and heat stimuli connection of memory filament, such smart fabric will allow generating massage effect (dynamic compression) using an external programmed heating source (Figure 4). ${ }^{6}$ No doubt, such memory textiles possess unique functions including stress and shape control abilities. These smart functions have the potential to overcome many challenges of conventional compression products, and could provide many novel solutions in the current treatment practice including customized stocking size development just before application, external pressure control and also mas- sage compression.

The availability of such memory textile with multi-functional benefits is the dream of all the related practitioners and manufacturer in the field. Herein, this dream seems to come true with the inception of smart memory fabric. Although the idea is working well at laboratory level but there are still several challenges need to meet including designing and optimization of memory textile before recommending it as a successful commercial venture.

\section{References}

1. Mosti G, Picerni P, Partsch H. Compression stockings with moderate pressure are able to reduce chronic leg oedema. Phlebology 2012;27:289-96.
2. Partsch H, Clark M, Mosti G, et al. Classification of compression bandages: practical aspects. Dermatol Surg 2008;34:600-9.

3. Kumar B, Hu JL, Pan N. Smart medical stocking using memory polymer for chronic venous disorders. Biomaterials 2016;75:174-81.

4. Kumar B, Hu J, Pan N. Memory bandage for functional compression management for venous ulcers. Fibers 2016;4:1.

5. Hu JL, Kumar B, Narayan HK. Stress memory polymers. J Polymer Sci Part B 2015;53:893-8.

6. Kumar B, Hu J, Pan N, Narayan HK. A smart orthopedic compression device based on a polymeric stress memory actuator. Mater Design 2016;97:222-9. 